\title{
A Study of Stress Analysis for a Residual Stress Model by Digital Photoelasticity
}

\author{
T. Kihara ${ }^{1, a}$ \\ ${ }^{1}$ Kinki University, School of Science and Engineering, Kowakae 3-4-1, Higashi-Osaka, Osaka, \\ Japan \\ a kihara@mech.kindai.ac.jp
}

Keywords: residual stress model, digital photoelasticity, phase shifting, phase unwrapping, three wavelengths.

\begin{abstract}
The stress analysis for a model with initial stresses, which we term a residual stress model, is performed by digital photoelasticity. The stresses applied on the residual stress model are obtained by analyzing both the initial stresses and the resultant stresses. The method used for analyzing the stresses applies the principle of superposition of the stress to photoelasticity, which is a well-known technique in the field of elasticity. In the digital photoelasticity technique used, the principal stress direction $\psi$ and the relative phase retardation $\rho$ are analyzed by photoelastic techniques using linearly polarized light. This technique overcomes the phase difference error associated with a quarter-wave plate by employing incident light at three different wavelengths, and using an unwrapping technique that allows $\psi$ and $\rho$ to be determined using the arctangent function. A residual stress model produced by a disk containing frozen stresses that was subjected to a diametral compressive load at an angle of $31^{\circ}$ was used to experimentally test this method. The values of the stresses of the loaded disk model analyzed were in good agreement with corre- sponding theoretical values at all locations far from the loading points of the residual stress model.
\end{abstract}

\section{Introduction}

Photoelasticity is an effective method for measuring the directions of principal stresses [ $\psi$ and $\psi+(\pi / 2)]$ and the difference of principal stresses, which is related to the relative phase retardation $\rho$, of a photoelastic model as visual patterns. The photoelastic model used is assumed to be free from the time-edge effect and from machining stresses, and initial stresses, which generate residual stresses. Developing such models is frequently a time-consuming, laborious process, and it requires a high degree of skill [1]. Since it is difficult to automate the production of models free from initial stresses, and it is desirable to perform stress analyses on models with initial stresses. Although such stress analyses are rare, they have been performed by determining the absolute value of $\rho$ for the loaded model by measuring the initial stresses and two resultant stresses generated when two different loads are applied [2]. It is anticipated that the applied stresses in a model with initial stresses can be determined by applying digital photoelasticity [3-12]. Digital photoelasticity can be used to measure $\psi$ and $\rho$ by using circularly polarized light [5,6], linearly polarized light [7,8], elliptically polarized light $[9,10]$ or white light $[11,12]$ as the incident light. 
The present paper describes a method for obtaining the applied stresses $\left(\sigma^{\prime}{ }_{\mathrm{x}}-\sigma^{\prime}{ }_{\mathrm{y}}, \tau^{\prime}{ }_{\mathrm{xy}}\right)$ on a residual stress model from the values of $\psi$ and $\rho$ for the initial and resultant stresses as measured by digital photoelasticity, where $\left(\sigma^{\prime}{ }_{\mathrm{x}}, \sigma^{\prime}{ }_{\mathrm{y}}, \tau^{\prime}{ }_{\mathrm{xy}}\right)$ are the rectangular stress components of the applied stresses. In the present technique, the values of $\psi$ and $\rho$ are measured using linearly polarized light. The analytical method for determining the stresses applies the principle of superposition of stresses to photoelasticity. The main premise of the paper is the employment of digital photoelasticity to tackle the problem of stress analysis for the residual stress model.

\section{Photoelastic Effects}

In a two-dimensional stress system, the principal stresses $\left(\sigma_{1}, \sigma_{2}\right)$ and principal stress direction $\psi$ are related to the rectangular stress components $\left(\sigma_{\mathrm{x}}, \sigma_{\mathrm{y}}, \tau_{\mathrm{xy}}\right)$ by the following equations [1]:

$$
\begin{aligned}
& \sigma_{1^{-}} \sigma_{2}=\left(\sigma_{x^{-}} \sigma_{\mathrm{y}}\right) / \cos 2 \psi=2 \tau_{\mathrm{xy}} / \sin 2 \psi, \\
& \psi=0.5 \tan ^{-1}\left\{2 \tau_{\mathrm{xy}} /\left(\sigma_{\mathrm{x}^{-}} \sigma_{\mathrm{y}}\right)\right\}
\end{aligned}
$$

If light propagates through a model with principal stresses $\left(\sigma_{1}, \sigma_{2}\right)$ and principal stress direction $\psi$, the relative phase retardation in optical lengths produced by double refraction is given by

$$
\rho_{\lambda \mathrm{i}}=\left(2 \pi t C_{\lambda_{\mathrm{i}}}\right)\left(\sigma_{1}-\sigma_{2}\right)
$$

where $\rho_{\lambda \mathrm{i}}$ is the relative phase retardation at $\lambda_{\mathrm{i}}$ (the wavelength of the light), $C_{\lambda \mathrm{i}}$ is the photoelastic sensitivity of the model at $\lambda_{\mathrm{i}}$, and $t$ is the thickness. The relation is known as the stress-optic law.

\section{Stresses Applied on Residual Stress Model}

When stresses $\left(\sigma_{1}^{\prime}, \sigma_{2}^{\prime}, \psi^{\prime}\right)$ are applied onto the residual stress model with the principal stresses ( $\sigma$ ${ }_{1}^{0}, \sigma^{0}{ }_{2}$ ) and principal stress direction $\psi^{0}$, a resultant stress field is produced in the model. Denoting the principal stresses of this resultant stress field by $\left(\sigma^{\prime 0}{ }_{1}, \sigma^{\prime 0}{ }_{2}\right)$, and the resultant principal stress direction by $\psi^{\prime 0}$, then the stress components of the residual stresses, the applied stresses, and the resultant stresses are given from Eq.(1). The applied stresses can be calculated by applying the principle of superposition of the stresses. Doing this gives the following expressions:

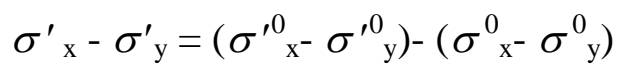

$$
\begin{aligned}
& =\left(\sigma^{\prime 0}{ }_{1}-\sigma^{\prime 0}{ }_{2}\right) \cos 2 \psi^{\prime \prime}-\left(\sigma^{0}{ }_{1}-\sigma^{0}{ }_{2}\right) \cos 2 \psi^{0} \text {, } \\
& \tau^{\prime}{ }_{\mathrm{xy}}=\tau^{\prime 0}{ }_{\mathrm{xy}}-\tau_{\mathrm{xy}}^{0} \\
& =0.5\left(\sigma^{\prime 0}{ }_{1}-\sigma^{\prime}{ }_{2}\right) \sin 2 \psi^{\prime 0}-0.5\left(\sigma^{0}{ }_{1}-\sigma^{0}{ }_{2}\right) \sin 2 \psi^{0} \text {. }
\end{aligned}
$$

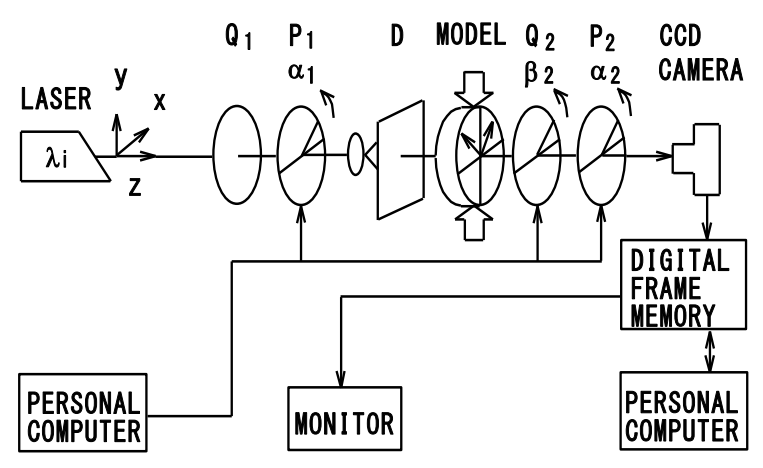

Fig. 1. Schematic diagram of an automatic whole-field measurement system using incident linearly polarized light: $\mathrm{P}_{1}$ and $\mathrm{P}_{2}$, linear polarizers; $\mathrm{Q}_{1}$ and $\mathrm{Q}_{2}$, quarterwave plates; $\mathrm{D}$, diffuser. 


\section{Photoelasticity Using Linearly Polarized Light}

The optical arrangement with wavelength $\lambda_{i}$ is shown in Fig. 1 . The linearly polarized light produced by polarizer $P_{1}$ impinges on the stressed model and passes through the analysis system, which consists of a quarter-wave plate $Q_{2}$ and a linear polarizer $P_{2}$. A quarter-wave plate $Q_{1}$ is used in order to reduce the change in intensity of the linearly polarized light produced by the rotating $P_{1}$. The light intensity $I\left(\alpha_{1}, \beta_{2}, \alpha_{2}\right)_{\lambda \mathrm{i}}$ emerging from $P_{2}$ can be represented as $[8,13]$

$$
\begin{aligned}
I\left(\alpha_{1}, \beta_{2}, \alpha_{2}\right) \lambda_{\mathrm{i}}=0.5 I_{0}\{1 & -2 \sin ^{2}\left(\rho_{\lambda_{\mathrm{i}}} / 2\right) \sin 2\left(\alpha_{1}-\psi\right)\left[\left(1+\sin \Delta \rho_{\lambda_{\mathrm{i}}}\right) \cos 2\left(\beta_{2}-\psi\right) \sin 2\left(\beta_{2}-\alpha_{2}\right)+\sin 2\left(\alpha_{2}-\psi\right)\right] \\
& -\left(1+\sin \Delta \rho_{\lambda_{\mathrm{i}}}\right) \sin 2\left(\beta_{2}-\alpha_{2}\right) \sin 2\left(\beta_{2}-\alpha_{1}\right)+\cos 2\left(\alpha_{2}-\alpha_{1}\right) \\
& \left.+\cos \Delta \rho_{\lambda_{\mathrm{i}}} \sin 2\left(\beta_{2}-\alpha_{2}\right) \sin \rho_{\lambda_{\mathrm{i}}} \sin 2\left(\alpha_{1}-\psi\right)\right\}+I_{\mathrm{N}}
\end{aligned}
$$

where,

$I_{0}=$ intensity of the incident light,

$I_{\mathrm{N}}=$ background intensity,

$\alpha_{1}=$ azimuth of the transmission axis of $P_{1}$,

$\alpha_{2}=$ azimuth of the transmission axis of $P_{2}$,

$\beta_{2} \square=$ azimuth of the fast axis of $\mathrm{Q}_{2}$,

$\Delta \rho_{\lambda \mathrm{i}}=$ phase difference error of $\mathrm{Q}_{2}$ to $\lambda_{\mathrm{i}}[14]$.

Using incident linearly polarized light of azimuth $\alpha_{1}$, the Stokes vector $\left\{S_{10}, S_{11}, S_{12}, S_{13}\right\}_{\lambda \mathrm{i}}$ from the model can be calculated from the light intensity $I\left(\alpha_{1}, \beta_{2}, \alpha_{2}\right) \lambda_{\mathrm{i}}$ measured by the following azimuth settings (azimuth values in degrees) of $\mathrm{Q}_{2}$ and $\mathrm{P}_{2}[8,15,16]$ :

$$
\left[\begin{array}{l}
S_{10} \\
S_{11} \\
S_{12} \\
S_{13}
\end{array}\right]_{\lambda i}=\left\{\begin{array}{c}
\left(S_{11}^{2}+S_{12}^{2}+S_{13}^{2}\right)^{0.5} \\
I(0,0,0)_{\lambda i}-I(0,90,90)_{\lambda i} \\
I(0,45,45)_{\lambda i}-I(0,135,135)_{\lambda i} \\
{\left[I(0,-45,0)_{\lambda i}-I(0,45,0)_{\lambda i}\right] / \cos \Delta \rho_{\lambda i}}
\end{array}\right\}
$$

Calculation of $\psi$ and $\rho_{\lambda \mathbf{i}}$. By substituting Eq. (6) into Eq.(7), the values of $\psi$ and $\rho_{\lambda \mathrm{i}}$ can be calculated using an arctangent function with abrupt jumps, as given by [8]

$$
\begin{aligned}
\psi & =0.25 \tan ^{-1}\left[\left(\mathrm{~s}_{12}+\mathrm{s}_{21}\right) \lambda_{\mathrm{i}} /\left(\mathrm{s}_{11^{-}}-\mathrm{s}_{22}\right) \lambda_{\mathrm{i}}\right] \\
& =0.25 \tan ^{-1}\left\{\left[2 \sin ^{2}\left(\rho_{\lambda \mathrm{i}} / 2\right) \sin 4 \psi\right] /\left[2 \sin ^{2}\left(\rho_{\lambda \mathrm{i}} / 2\right) \cos 4 \psi\right]\right\} \quad \text { for } \rho_{\lambda \mathrm{i}} \neq 2 \pi N_{\lambda \mathrm{i}}, \\
\rho_{\lambda \mathrm{i}} & =\tan ^{-1}\left[\left(\mathrm{~s}_{13 \lambda \mathrm{i}} \sin 2 \psi-\mathrm{s}_{23} \lambda_{\mathrm{i}} \cos 2 \psi\right) /\left(\mathrm{s}_{11}+\mathrm{s}_{22}-1\right) \lambda_{\mathrm{i}}\right]=\tan ^{-1}\left[\sin \rho_{\lambda \mathrm{i}} / \cos \rho_{\lambda \mathrm{i}}\right] .
\end{aligned}
$$

where $\left\{s_{10}, s_{11}, s_{12}, s_{13}\right\}_{\lambda \mathrm{i}}$ denotes the normalized Stokes vector $\left\{S_{10} / S_{10}, S_{11} / S_{10}, S_{12} / S_{10}, S_{13} / S_{10}\right\}_{\lambda \mathrm{i}}$ at $\lambda_{\mathrm{i}}$. In this system, $\psi$ and $\rho_{\lambda \mathrm{i}}$ are not affected by the phase difference error $\Delta \rho_{\lambda \mathrm{i}}$ of $Q_{2}$ for an arbitrary wavelength $\lambda_{\mathrm{i}}[16]$.

\section{Determination of $\psi$ and $\rho_{\lambda \text { itot }}$ using Three Wavelengths Calculation}

Determination of $\psi$. By using Eq. (8), $\psi$ can be obtained in the range $(-\pi / 8, \pi / 8)$, except at the points when the total relative phase retardation $\rho_{\lambda \text { itot }}=2 \pi N_{\lambda \mathrm{i}}\left(N_{\lambda_{\mathrm{i}}}\right.$ : integer $)$ at $\lambda_{\mathrm{i}}$. The values of $\psi$ can be measured more accurately when the fractional value $\rho_{\lambda \mathrm{i}}$ is larger. Therefore, $\psi$ can be determined from Eq. (10) by using images obtained at three appropriately selected wavelengths $\left(\lambda_{1}, \lambda_{2}, \lambda_{3}\right)$, 


$$
\begin{aligned}
\psi= & 0.25 \tan ^{-1}\left\{\left[\left(\mathrm{~s}_{12}+\mathrm{s}_{21}\right)_{\lambda 1}+\left(\mathrm{s}_{12}+\mathrm{s}_{21}\right)_{\lambda 2}+\left(\mathrm{s}_{12}+\mathrm{s}_{21}\right){ }_{\lambda 3}\right] /\left[\left(\mathrm{s}_{11^{-}}-\mathrm{s}_{22}\right) \lambda_{1}+\left(\mathrm{s}_{11^{-}} \mathrm{s}_{22}\right)_{\lambda 2}+\left(\mathrm{s}_{11^{-}} \mathrm{s}_{22}\right)_{\lambda 3}\right]\right\} \\
= & 0.25 \tan ^{-1}\left\{\left\{\left[\sin ^{2}\left(\rho_{\lambda 1} / 2\right)+\sin ^{2}\left(\rho_{\lambda 2} / 2\right)+\sin ^{2}\left(\rho_{\lambda 3} / 2\right)\right] 2 \sin 4 \psi\right\}\right. \\
& \left./\left\{\left[\sin ^{2}\left(\rho_{\lambda 1} / 2\right)+\sin ^{2}\left(\rho_{\lambda 2} / 2\right)+\sin ^{2}\left(\rho_{\lambda 3} / 2\right)\right] 2 \cos 4 \psi\right\}\right\},
\end{aligned}
$$

where, $\left(\mathrm{s}_{12}+\mathrm{s}_{21}\right) \lambda_{\mathrm{i}}$ and $\left(\mathrm{s}_{11}-\mathrm{s}_{22}\right) \lambda_{\mathrm{i}}$ denote $\left(\mathrm{s}_{12}+\mathrm{s}_{21}\right)$ and $\left(\mathrm{s}_{11}-\mathrm{s}_{22}\right)$ obtained at $\lambda_{\mathrm{i}}$, respectively.

This method for determining $\psi$ is able to compensate for the influence of $\rho_{\lambda \text { itot }}=2 \pi N_{\lambda_{\mathrm{i}}}$ except for $N_{\lambda \mathrm{i}}=0$. The measurement range of $\psi$ can be extended to $(-\pi / 2, \pi / 2)$. The unwrapping process is performed by adding a constant value $\pi / 4$ or $-\pi / 4$ to eliminate the abrupt jumps, $\pi / 4$ or $-\pi / 4$, in $\psi$.

Determination of $\rho_{\lambda \text { itot }}$. The values of $\rho_{\lambda \mathrm{i}}$ are calculated from Eq. (9) in the range $(-\pi / 2, \pi / 2)$ for the known mode of $\pi$. The total relative phase retardation $\rho_{\lambda \text { itot }}$ can be found using the calculated $\rho_{\lambda \mathrm{i}}$ if $\rho_{\lambda \text { itot }}$ is continuous. In the same way as the unwrapping process for $\psi$, the unwrapping process for $\rho_{\lambda \mathrm{i}}$ eliminates phase jumps $(-\pi / 2, \pi / 2)$ in $\rho_{\lambda \mathrm{i}}$ so that $\rho_{\lambda \mathrm{i}}$ becomes continuous. Then, $\rho_{\lambda \text { itot }}$ can be determined by adding a constant value to the continuous $\rho_{\lambda \mathrm{i}}$. When $\rho_{\lambda \text { itot }}$ at a point on the model is known, the constant value at this point can be found by comparing $\rho_{\lambda \text { itot }}$ with the continuous $\rho_{\lambda i}$. The value of $\rho_{\lambda \text { itot }}$ can also be found from the integer $N_{\lambda_{\mathrm{i}}}$ and the fractional value $\rho_{\lambda_{\mathrm{i}}}$ [8].

\section{Experiments and Discussion}

For the purposes of determining $\psi$ and $\rho_{\lambda \text { itot }}$, three different wavelengths used should be selected so as to produce fractional values that have large differences in the measurement range of the model. In this study, the wavelengths $\lambda_{1}=636.0 \mathrm{~nm}, \lambda_{2}=537.8 \mathrm{~nm}$ and $\lambda_{3}=441.6 \mathrm{~nm}$ were used by using laser. The phase difference errors of $\mathrm{Q}_{2}$ for $\lambda_{1}, \lambda_{2}, \lambda_{3}$ were $\Delta \rho_{\lambda 1}=-17.2^{\circ}, \Delta \rho_{\lambda 2}=-3.9^{\circ}$ and $\Delta \rho_{\lambda 3}=14.9^{\circ}$, respectively. However, it is important to note that $\psi$ and $\rho_{\lambda \mathrm{i}}$ determined from Eqs. (8), (9) are unaffected by $\Delta \rho_{\lambda i}$.

The model used was an epoxy resin circular disk (diameter $2 R=40.0 \mathrm{~mm}$, thickness $t=6.0 \mathrm{~mm}$, $\left.C_{\lambda 1}=0.0774 \mathrm{~mm} / \mathrm{N}, C_{\lambda 2}=0.0954 \mathrm{~mm} / \mathrm{N}, C_{\lambda 3}=0.1253 \mathrm{~mm} / \mathrm{N}\right)$. The initial stresses of the model that produced the residual stresses were created by employing a frozen stress disk under a diametral compressive load, as illustrated in Fig. 2. Actual stresses produced by a vertical diametral compressive load of $P=198 \mathrm{~N}$ are then applied on this initial frozen stress model. Figures 3(A) and (B) show the measured images for the initial frozen stress model and the resultant stress model,

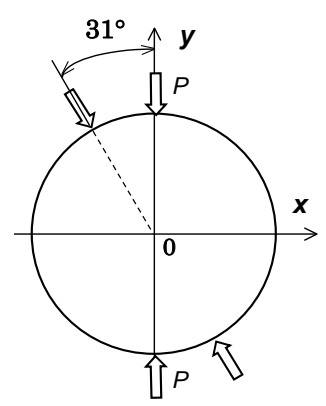

Fig. 2. The initial frozen stresses and the applied stresses: The initial frozen stresses are produced by a diametral compressive load applied at an angle of $31^{\circ}$, and the applied stresses are produced by a vertical diametral compressive load.

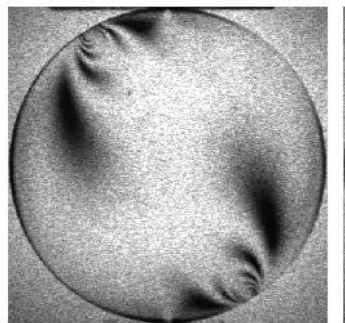

(A)

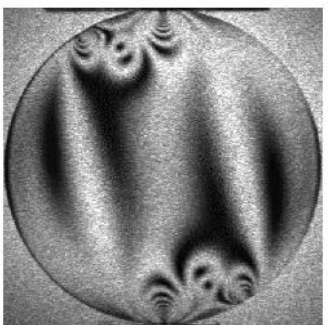

(B)
Fig. 3. (A) and (B) show image $I^{0}(45,135$, $135)_{\lambda 2}$ for the initial frozen stress model, and image $I^{\prime 0}(45,135,135)_{\lambda 2}$ for the resultant stress model, respectively. 


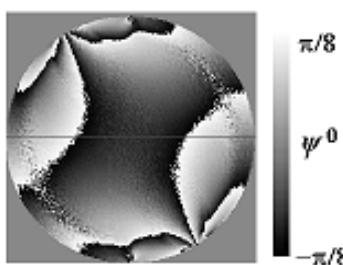

(A)

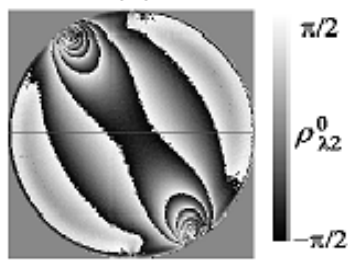

(D)

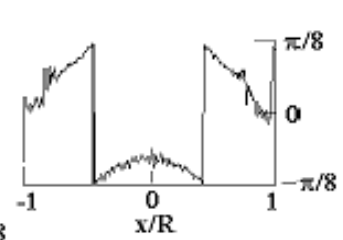

(B)

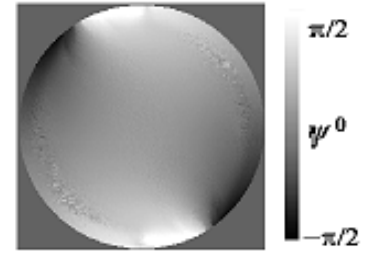

(C)

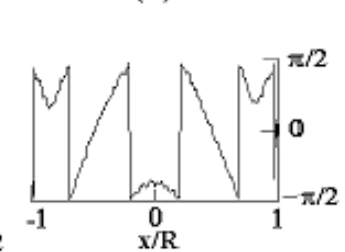

(E)

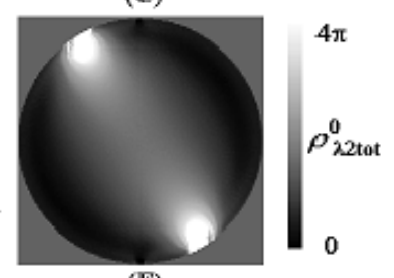

(F)

Fig.4. Analysis of $\psi^{0}$ and $\rho_{\lambda 2}{ }^{0}$ tot for the initial frozen stress model. (A) $\psi^{0}$ obtained by using three wavelengths, (B) distribution of $\psi^{0}$ in the horizontal diametral direction (C) unwrapped $\psi^{0}$, (D) image of $\rho_{\lambda 2}{ }^{0}$, (E) distribution of $\rho_{\lambda 2}{ }^{0}$ in the horizontal diametral direction, and (F) image of $\rho_{\lambda 2}{ }^{0}$ tot.

respectively. The image in Fig. 3(B) displays fringes due to both the initial frozen stresses and the actual stresses produced by the applied stresses.

The $\psi^{0}$ for the initial frozen stress model was calculated from Eq. (10) using three wavelengths, since $\psi^{0}$ cannot be measured in the neighborhood of positions for which $\rho_{\lambda \mathrm{i} \text { tot }}{ }^{0}=2 \pi N_{\lambda_{\mathrm{i}}}$ at $\lambda_{\mathrm{i}}$. The calculated $\psi^{0}$ is shown in Figs. 4(A) and (B), where the range of $\psi^{0}$ is $[-\pi / 8, \pi / 8]$. Then, $\psi^{0}$ can be unwrapped to extend this range to $[-\pi / 2, \pi / 2]$ and the unwrapped results are shown in Fig. 4(C). It should be noted that $\psi$ is not measured at the position $\rho_{\text {tot }}=0$, and the measurement of $\psi$ near $\rho_{\text {tot }}=0$ is poor; consequently, there are sand type errors for $\psi$ in Figs. 4(A)-(C). Nevertheless, the calculated $\psi^{0}$ was well compensated by using three wavelengths. The image and distribution of $\rho_{\lambda 2}{ }^{0}$ for the initial frozen stress model is shown in Figs. 4(D) and (E). These figures show that the values of $\rho_{\lambda 2}{ }^{0}$ were hardly influenced by the sand type errors in $\psi$. Since the retardation on the model changes continually, $\rho_{\lambda 2}{ }^{0}$ was connected by eliminating the phase jumps, $\pi$ or $-\pi$, from the center of the disk. Figure 4(F) shows the values of $\rho_{\lambda 2}{ }^{0}$ tot obtained by adding the constant value $2 \pi$ to the continuous $\rho_{\lambda 2}{ }^{0}$. Similarly, images of $\psi^{\prime 0}$, unwrapped $\psi^{\prime 0}, \rho_{\lambda 2^{\prime}}{ }^{0}$ and unwrapped $\rho_{\lambda 2^{\prime}}{ }^{0}$ tot for the resultant stress model are shown in Figs.

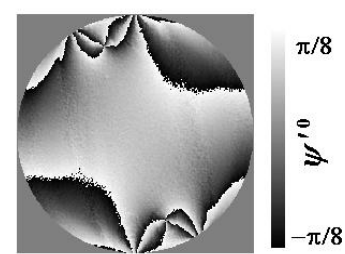

(A)

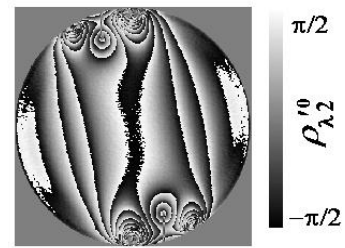

(C)

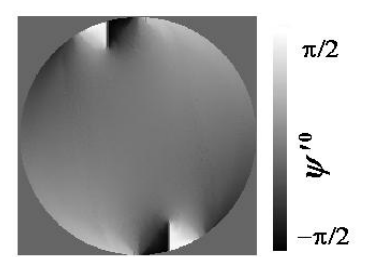

(B)

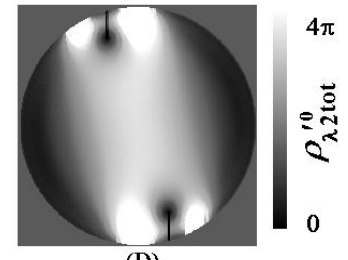

(D)
Fig. 5. Analysis of $\psi^{\prime 0}$ and $\rho_{\lambda 2^{\prime}}{ }^{0}$ tot for the resultant stress model: (A) $\psi^{\prime 0}$ obtained by using three wavelengths, (B) unwrapped $\psi^{\prime 0}$, (C) image of $\rho_{\lambda 2^{\prime}}$, and (D) image of $\rho_{\lambda 2}{ }^{{ }^{0}}$ tot.

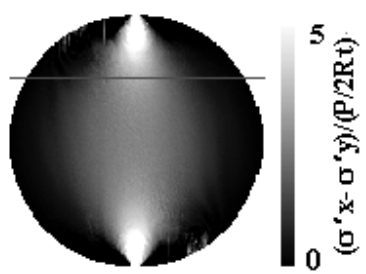

(A)

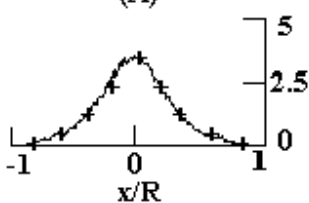

(B)

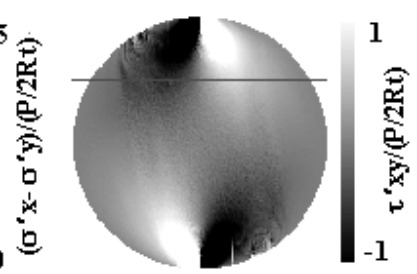

(C)

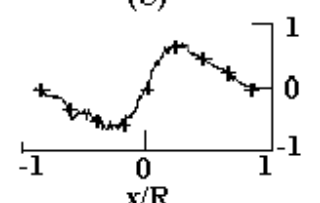

(D)
Fig. 6. $\left(\sigma^{\prime}{ }^{-}-\sigma^{\prime}\right)$ and $\tau_{x y}^{\prime}$ for the applied

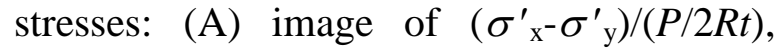
while (B) the distribution of $\left(\sigma^{\prime}{ }^{\prime}-\sigma^{\prime}{ }_{y}\right)$ $/(P / 2 R t)$ along the horizontal axis at $\mathrm{y}=0.5 R$, where the ' + ' symbols show the corresponding theoretical values. (C) image of $\tau^{\prime}{ }_{x y}$ $/(P / 2 R t)$, while (D) the distribution at $y=0.5 R$. 
5(A)-(D), respectively.

The values of $\left(\sigma^{\prime}{ }_{x}-\sigma^{\prime}{ }_{y}\right)$ and $\tau^{\prime}{ }_{x y}$ of the actual applied stresses are calculated from Eqs. (4) and (5), respectively. The images of dimensionless stresses $\left(\sigma^{\prime}{ }^{\prime}-\sigma^{\prime}{ }_{y}\right) /(P / 2 R t)$ and $\tau^{\prime}{ }_{x y} /(P / 2 R t)$ are shown in Figs. 6(A) and (C). Figures 6(B) and (D) show respectively the distributions of these stresses along the horizontal axis at $\mathrm{y}=0.5 R$, and theoretically obtained values [17] denoted by the ' + ' symbols are also shown in both plots. Clearly the values for these two quantities obtained experimentally by the present method are in good agreement with the theoretical values everywhere except in the neighborhoods of the loading points in the residual stress model.

\section{Conclusions}

Stress analysis for a model with initial stresses was performed by digital photoelasticity using linearly polarized light. The actual applied stresses $\left(\sigma^{\prime}{ }_{x}-\sigma^{\prime}{ }_{y}\right)$ and $\tau_{x y}^{\prime}$ on the residual stress model are obtained by analyzing both the initial stresses and the resultant stresses. The method for analyzing the stresses applied the principle of superposition of stresses to the photoelasticity technique. In a residual stress model produced by applying diametral compressive loads to a disk containing frozen stresses, the values of $\left(\sigma^{\prime}{ }_{x}-\sigma_{y}^{\prime}\right)$ and $\tau^{\prime}{ }_{x y}$ obtained experimentally by the present method were found to be in good agreement with corresponding theoretical values everywhere except in the neighborhoods of the loading points in the residual stress model.

\section{References}

[1] M.M. Frocht: Photoelasticity Vol.1, John Wiley \& Sons (1949).

[2] J.-G. Suh, J.-S. Hawong and S.-H. Choi: Trans. Korean Soc. Mech. Eng. (in Korean), Vol. 19 (1995), p.1190-1201.

[3] K. Ramesh: Digital Photoelasticity, Springer-Verlag (2000).

[4] A.D. Nurse: Applied Optics, Vol. 36 (1997), p.5781-5786.

[5] T. Kihara: Proc. of the Japan Society for Photoelasticity (in Japanese), Vol. 9 (1989), p.1-8.

[6] E.A. Patterson, and Z. F. Wang: Strain, Vol.(1991), p.49-56.

[7] T. Kihara: Proc. of 9th Int. Conf. Exp. Mech. 2,Copenhagen, (1990), p.821-827.

[8] T. Kihara: Strain, Vol. 39 (2003), p.65-71.

[9] S.Yoneyama, and M. Takashi: JSME International Journal, Series A, Vol. 42 (1999), p.396-402.

[10] T. Kihara: Strain, Vol.42 (2006), p.255-263.

[11] A.Ajovalasit, S. Barone, and G. Petrucci: Exp. Mech., Vol. 35 (1995), p.193-200.

[12] G.. Petrucci, Exp. Mech., Vol. 37 (1997), p.420-426.

[13] D. Clarke and J.F. Grainger: Polarized Light and Optical Measurement, Pergamon Press, Oxford (1971).

[14] S. Barone, G. Burriesci, and G. Petrucci: Exp. Mech., Vol. 42 (2002), p.132-139.

[15] T. Kihara: Optics Comm., Vol. 110 (1994), p.529-532.

[16] T. Kihara: Advance in Experimental Mechanics IV (J.M. Dulieu-Barton and S. Quinn, ed.), Trans Tech Publications, Switzerland, (2005), p.235-240.

[17] M.M. Frocht: Photoelasticity Vol.2, Chap.4, John Wiley \& Sons (1948). 